\title{
Acknowledgment to Reviewers of JMMP in 2020
}

\section{JMMP Editorial Office}

MDPI AG, St. Alban-Anlage 66, 4052 Basel, Switzerland

Citation: JMMP Editorial Office.

Acknowledgment to Reviewers of JMMP in 2020. J. Manuf. Mater.

Process. 2021, 5, 14. https://doi.org/ 10.3390/jmmp5010014

Published: 28 January 2021

Publisher's Note: MDPI stays neutral with regard to jurisdictional claims in published maps and institutional affiliations.

Copyright: (C) 2021 by the author. Licensee MDPI, Basel, Switzerland. This article is an open access article distributed under the terms and conditions of the Creative Commons Attribution (CC BY) license (http:// creativecommons.org/licenses/ by/4.0/).

Peer review is the driving force of journal development, and reviewers are gatekeepers who ensure that JMMP maintains its standards for the high quality of its published papers. Thanks to the cooperation of our reviewers, in 2020, the median time to first decision was 15 days and the median time to publication was 32 days. The editors would like to express their sincere gratitude to the following reviewers for their precious time and dedication, regardless of whether the papers were finally published:

Agiasofitou, Eleni

Alaluss, Khaled

Alar, Vesna

Alcarria, Ramon

Andriollo, Tito

Antosz, Katarzyna

Arevalo, Cristina

Axinte, Eugen

Azizi, Aydin

Bähre, Dirk

Bai, Mingwen

Balle, Frank

Barbi, Silvia

Barriobero-Vila, Pere

Bartkowska, Aneta

Bartnicki, Jarosław

Bellantone, Vincenzo

Benardos, Panorios

Bengtsson, Marcus

Benhassine, Mehdi

Bernardo, Luis Filipe Almeida

Bidulská, Jana

Biswal, Romali

Blaga, Lucian A.

Blum, Wolfgang

Boccarusso, Luca

Bombac, David

Bordeenithikasem, Punnathat

Boroński, Dariusz

Borsato, Milton

Bou, Santiago Ferrandiz

Bozena, Gajdzik

Brandão, Lincoln Cardoso

Buchmayr, Bruno

Buhl, Johannes

Calaf Chica, Jose
Calvo, Roque

Camelio, Jaime

Campos Rubio, Juan Carlos

Cantero Guisández, José Luis

Cao, Pingxiang

Cardon, Ludwig

Carou, Diego

Cerchione, Roberto

Ceruti, Alessandro

Chang, Tai-Woo

Chaudhari, Akshay

Chaus, Alexander S.

Chicos, Lucia-Antoneta

Cho, Sunghun

Cicero, Sergio

Cinkilic, Emre

Correa, Diego Rafael Nespeque

Costantino, Francesco

Cuan-Urquizo, Enrique

Da Silva, Francisco José Gomes

De Freitas Daudt, Natália

De Lacalle, Luis N. López

de Souza, Adriano Fagali

Deja, Mariusz

Demo, Pavel

Dhamdhere, AJIT

Diaz, Jose

Diniz, Anselmo

Dixon, David A.

Dobrocky, David

Dong, Yan

Dorado, Ruben

Duchosal, Arnaud

Dulska, Agnieszka

Durejko, Tomasz

Egan, Paul F. 
Eitzinger, Christian

Eliseev, Alexander

Elkaseer, Ahmed

ElSayed, Ahmed

Epp, Jeremy

Everett, Richard

Feito, Norberto

Feng, Yixuan

Fernandez-Vidal, Severo Raul

Fragassa, Cristiano

França, Thiago Valle

Francalanza, Emmanuel

Fujii, Yoshihisa

Fydrych, Dariusz

Galkiewicz, Jaroslaw

Galvão, Ivan

Gao, Liang

Garašić, Ivica

García, Marcelo

Gavrus, Adinel

Ghaffar, Seyed

González Pérez, Isaías

González, Hernán

Grabowska, Sandra

Grützmacher, Philipp

Guerrero-Vaca, Guillermo

Ha, Jinjin

Habrat, Witold

Hamel, William R.

Hassan, Mahmoud

Hassui, Amauri

Hayakawa, Tohru

He, Binbin

He, Yihai

Hee, Ay Ching

Hegab, Hussien

Hełdak, Maria

Helen Margaret, Davies

Hertweck, Benjamin

Hidalgo, Javier

Hildebrand, Joerg

Holub, Michal

Höppel, Heinz Werner

Hovanski, Yuri

Hsia, Shao-Yi

$\mathrm{Hu}$, Yuh-Chung

Huo, Qinghuan

Hwangbo, Hyunwoo

Ihara, Yukitoshi

Ikram, Awais

Isanaka, Sriram Praneeth
Ito, Kazuhiro

Ito, Kiyohiro

Jackson, Mark

Jandaghi, Mohammad Reza

Jasiewicz, Marcin

Jerez-Mesa, Ramón

Jia, Fanghui

Jin, Xie

Jin, Yifei

Kaczmarczyk, Jarosław

Kamiński, Marcin

Kamiya, Osamu

Kampa, Adrian

Karg, Michael C. H.

Karlík, Miroslav

Karnati, Sreekar

Kazmer, David O.

Kechagias, John

Kechagias, John D.

Kellens, Karel

Kim, Man-Hoe

Kitagawa, Jiro

Kizaki, Toru

Kłonica, Mariusz

Kminiak, Richard

Koblischka, Michael R.

Koike, Mari

Konda Gokuldoss, Prashanth

Konovalov, Sergey

Kordoš, Marcel

Kowalczyk, Małgorzata

Kozakiewicz, Anna

Krejcar, Ondrej

$\mathrm{Ku}$, Tae-Wan

Kubit, Andrzej

Kuczmaszewski, Jozef

Kuhn, Erik

Kukla, Christian

Kulawik, Adam

Kumar, Ch Sateesh

Kunetsov, Vladimir

Kurfess, Thomas

Kyratsis, Panagiotis

Ladani, Leila Jannesari

Lancaster, Robert

Lee, Sang Jin

Lee, Sun-Kyu

Lee, Yooneun

Legutko, Stanisław

Lehotzky, David

Lei, Shuting 
Lermen, Richard Thomas

Li, Anhai

Li, Duo

Li, Qing

$\mathrm{Li}$, Tao

Li, Yang

Lian, Yunsong

Liedl, Gerhard

Lim, Jiseok

Liu, Changqing

Liu, Dabiao

Liu, Tian

Liu, Wei

Lontos, Antonios

Lorusso, Massimo

$\mathrm{Lu}$, Xiaohong

Lunt, Alexander J.G.

Luo, Liang

Luo, Xichun

Ma, Xiang

Manolakos, Dimitrios

Mansour, Gabriel

Markdahl, Johan

Markopoulos, Angelos P.

Martínez López, José Israel

Maru, Marcia M.

Maruda, Radosław W.

Masato, Davide

Mazzucato, Federico

McMillan, Alison J.

Mcnelley, Terry R.

Meenashisundaram, Ganesh Kumar

Mia, Mozammel

Michalcová, Alena

Mikolajczyk, Tadeusz

Milichovský, František

Miller, Scott F.

Minguella-Canela, Joaquim

Mirone, Giuseppe

Molaei, Reza

Mroczka, Krzysztof

Mucha, Jacek

Mukherjee, Tuhin

Müller, Julian Marius

Mun, Sang Don

Nadolny, Krzysztof

Nakagawa, Yuki

Nambu, Koichiro

Nassiri, Ali

Nee, Andrew

Nejman, Alicja
Nemeş, Ovidiu

Niemczewska-Wojcik, Magdalena

Niemczewska-Wójcik, Magdalena

Nikolova, Maria

Okechukwu, Okorie

Okunkova, Anna A.

Oliveira, Monica

Öpöz, Tahsin

Otto, Andreas

Ozerov, Maxim

Pahlevani, Farshid

Pamuković, Jelena Kilić

Pankowska, Malgorzata

Pantazopoulos, George A.

Patel, Vivek

Pater, Zbigniew

Patterson, Albert E.

Pekarcikova, Marcela

Pellizzeri, Tiffany

Piccininni, Antonio

Pilipović, Ana

Polasik, Robert

Politis, Denis

Popa, Florin

Pradhan, Kallol

Pruncu, Catalin I.

Racineux, Guillaume

Radev, Dimitar

Radonic, Vasa

Rahman, Muhommad Azizur

Raileanu, Silviu

Ribeiro, Carlos A. Silva

Rivière-Lorphèvre, Edouard

Rosenkranz, Andreas

Rosso, Mario

Sakon, Takuo

Salguero, Jorge

Salmi, Mika

Salonitis, Konstantinos

Salvati, Enrico

Sánchez, Antonio

Sanchis, Raquel

Sarosi, Codruta

Sarvestani, Hamidreza Yazdani

Sato, Ryuta

Schiopu, Ionut

Schönemann, Lars

Schoop, Julius

Seong, Woo-Jae

Seriani, Stefano

Shaburova, Nataliya 
Shala, Ahmet

Shalaby, Mohamed

Shao, Guodong

Shih, Ching-long

Shimizu, Yuki

Shishkin, Andrei

Shokrani, Alborz

Sili, Andrea

Silva, Francisco

Sing, Swee Leong

Skripnyak, Vladimir A.

Slatineanu, Laurentiu

Slobodyan, Mikhail

Ślusarczyk, Beata

Smoleń, Andrzej

Sofia, Daniele

Šoltysová, Zuzana

Somireddy, Madhukar

Sortino, Marco

Speranzini, Emanuela

Squillace, Antonino

Stachiv, Ivo

Stanciu, Mariana Domnica

Stavropoulos, Panagiotis

Stern, Adin

Stetter, Ralf

Struzikiewicz, Grzegorz

Šugár, Peter

Surzhenkov, Andrei

Svaco, Marko

Świercz, Rafał

Świerczyńska, Aleksandra

Szczucka-Lasota, Bożena

Szwajka, Krzysztof

Tan, Caiwang

Tanaka, Satoshi

Tarasov, Sergei Yu

Tavares, S.M.O.

Thapliyal, Vivek

Thiede, Sebastian

Tofangchi, Alireza

Tomáš, Miroslav

Tournier, Christophe

Trotta, Gianluca

Trzepiecinski, Tomasz

Tsai, Meng-Hsiu

Tsai, Meng-Shiun

Tsao, Chung Chen

Unglaub, Julian

Urbikain, Gorka
Vaitkus, Saulius

Valerga Puerta, Ana Pilar

Van Den Boogaard, A.H.

Vazquez-Martinez, Juan M.

Venuti, Valentina

Vergara, Diego

Victor J., Rico

Vidakis, Nectarios

Vivek, Anupam

Voloshin, Aleksandr

Volpp, Joerg

Vosniakos, George-Christopher

Voznyak, Yuri

Wachowski, Marcin

Wang, Baicun

Wang, Junfeng

Wang, Sibao

Wang, Tianhao

Wang, Xiaoqing

Wenzel, Tobias

Wie, Jeong Jae

Winczek, Jerzy

Wiora, Józef

Wojciechowski, Szymon

Worrall, Stephen

Wu, Mingtao

Xie, Haibo

Yang, Shuming

Yang, Zhensheng

Yang, Zhibo

Yeh, Syh-Shiuh

Yeo, Zhiquan

Yeong, Wai Yee

Yin, Fei

Yu, Haiping

Yu, Nan

Yusenko, Kirill V.

Zarrintaj, Payam

Zawada-Tomkiewicz, Anna

Zeljkovic, Milan

Zhang, Chaoqun

Zhang, Hao

Zhang, Julie

Zhang, Shenli

Zhang, Yan

Zhang, Yang

Zhang, Yijie

Zhou, Peng

Zhu, Lin 